\title{
Scattering of a dyon from hydrogen atom
}

\author{
P.C. Pant, V.P. Pandey and B.S. Rajput ${ }^{*}$ \\ Department of Physics, Kumaun University, Nainital-263 002, India
}

\begin{abstract}
Undertaking the scattering of a dyon from a hydrogen atom it has been demonstrated that scattering cross section is perceptibly modified from the scattering cross section of scattering of an electron from a hydrogen atom due to the presence of magnetic charge on dyon and high energy of dyon involved in the scattering process.
\end{abstract}

\section{INTRODUCTION}

Physicists have long been interested in the existence of magnetic monopole. The early historical interest in monopoles was due to the symmetry between electric and magnetic fields in Maxwell's equation. However, due to lack of abundance of free magnetic charge compared to electric charges they were not included in the final formulation of those equations. In 1931 Dirac $^{1}$ showed that existence of free magnetic charge (Dirac monopole) could provide reason for quantization of electric charge ${ }^{2}$. This work motivated renewed interest in searching for monopoles. Although there was no guidance as to the mass, size, etc. of these monopoles several experimental consequences were apparent.

It was assumed that the monopole mass would not be very much different from other elementary particles (e. g. protons) and would be highly relativistic. As such these

\footnotetext{
* Corresponding author
} 
would produce a great deal of ionization while passing through matter but none of these effects were observed and literature turned partially negative casting doubt on the existence of these particles.

A fresh interest in the subject was enhanced when 't. Hooft ${ }^{3}$ and Polyakov ${ }^{4}$ demonstrated separately that monopoles exist as solutions in many non-Abelian gauge theories. The possibility of these GUT monopoles provide stimulus for much recent interest in the subject. These monopoles have enormous importance in connection with the problem of quark confinement ${ }^{5}$ of quantum chromodynamics, C.P. violation ${ }^{6}$, Proton decay $^{7}$ and baryon number non-conservation processes ${ }^{8}$. Inspite of potential importance of these particles the theories to describe them suffered from many paradoxes such as Dirac's veto and wrong connection between spin and statistics ${ }^{9}$. Schwinger ${ }^{10}$ showed that some of these problems can be resolved by taking electric and magnetic charge on the same particle known as dyon. Moreover, Witten has shown that monopoles are necessarily dyons ${ }^{6}$.

The theories to describe these particles were also clumsy and manifestly noncovariant. In order to develop a theory for these particles which will be conceptually as transparent as the usual quantum electrodynamics is, we ${ }^{11,12,13}$ started with the idea of two four-potentials to avoid the use of singular potential by taking generalized charge, generalized four potential, generalized four-current associated with these particles as complex quantities with their real and imaginary parts as electric and magnetic constituents. With the help of this theory we have undertaken the study of bound states and scattering of dyon-dyon ${ }^{14}$ and dyon-fermion ${ }^{15}$ systems and it has been demonstrated that exact solutions of bound states for these system in relativistic frame work is not possible due to the presence of a term vanishing more rapidly than $r^{-1}$ in the potential of such system. To overcome this difficulty we studied the Pauli equation for dyon-dyon ${ }^{16}$ and dyon-fermion ${ }^{17}$ system by adhoc introduction of spin in the Hamiltonian of the system and obtained bound state solutions in Abelian and non-Abelian gauge theories.

We have further studied the bound states of three and four dyons ${ }^{18,19}$ and have demonstrated that the bound state solutions are quite modified from the bound state solutions of quantum electrodynamics due to the presence of magnetic charge on dyon. Extending this work in the present paper we have undertaken the problem of scattering of an energetic dyon from a Hydrogen atom and it has been demonstrated that scattering cross section is perceptibly modified from the usual scattering cross section of a fermion from Hydrogen atom due to the presence of magnetic charge on dyon. 


\section{SCATTERING OF A DYON FROM A HYDROGEN ATOM}

In order to undertake the scattering of a dyon from a Hydrogen atom we assume that atom has infinite degrees of freedom, so that it can be excited during the scattering process. The incident dyon may change place with the electron of atom and hence exchange effects may occur in the collision. The incident dyon produces generalized electromagnetic field which may polarize the target atom and hence polarization effects are also involved .

If we consider the energy of incident dyon as very high the exchange and the polarization effects are unimportant and can be left out of consideration.

The Hamiltonian for describing the scattering of a dyon by a Hydrogen atom may be written as

$$
\hat{\mathrm{H}}=\hat{\mathrm{H}}_{\mathrm{o}}+\hat{\mathrm{H}}^{\prime}
$$

where $\hat{\mathrm{H}}_{\mathrm{o}}=-\frac{\hbar^{2}}{2 \mathrm{~m}} \hat{\nabla}_{1}^{2}-\frac{\hbar^{2}}{2 \mathrm{~m}} \hat{\nabla}_{2}^{2}-\frac{\mathrm{e}^{2}}{\mathrm{r}_{1}}$

describes the internal motion of the atom together with the kinetic energy of the relative motion of the incident dyon and the scatterer atom and

$$
\hat{\mathrm{H}}^{\prime}=-\frac{\mathrm{e}^{2}}{\mathrm{r}_{12}}+\frac{(\mathrm{eg})^{2}}{2 \mathrm{mr}_{12}^{2}}-\frac{\mathrm{e}^{2}}{\mathrm{r}_{2}}+\frac{(\mathrm{eg})^{2}}{2 \mathrm{mr}_{2}^{2}}
$$

represents the interaction between the incident particle and the scatterer. Hence equation (2.1) can be written as

$$
\hat{\mathrm{H}}=-\frac{\hbar^{2}}{2 \mathrm{~m}} \hat{\nabla}_{1}^{2}-\frac{\hbar^{2}}{2 \mathrm{~m}} \hat{\nabla}_{2}^{2}-\frac{\mathrm{e}^{2}}{\mathrm{r}_{1}}-\frac{\mathrm{e}^{2}}{\mathrm{r}_{12}}+\frac{(\mathrm{eg})^{2}}{2 \mathrm{mr}_{12}^{2}}-\frac{\mathrm{e}^{2}}{\mathrm{r}_{2}}+\frac{(\mathrm{eg})^{2}}{2 \mathrm{mr}_{2}^{2}}
$$

The eigenfunctions of $\hat{\mathrm{H}}_{\mathrm{O}}$ are specified by two parameter

$$
\hat{\mathrm{H}}_{\mathrm{o}} \phi_{\alpha \mathrm{a}}\left(\overrightarrow{\mathrm{r}}_{1}, \overrightarrow{\mathrm{r}}_{2}\right)=\mathrm{E}_{\alpha \mathrm{a}} \phi_{\alpha \mathrm{a}}\left(\overrightarrow{\mathrm{r}}_{1}, \overrightarrow{\mathrm{r}}_{2}\right)
$$

Here $\alpha$ - specifies the initial quantum state of the incident dyon and a-specifies that of the atom. We can write

$$
\phi_{\alpha \mathrm{a}}\left(\overrightarrow{\mathrm{r}}_{1}, \overrightarrow{\mathrm{r}}_{2}\right)=\mathrm{w}_{\mathrm{a}}\left(\overrightarrow{\mathrm{r}}_{1}\right) \phi_{\alpha}\left(\overrightarrow{\mathrm{r}}_{2}\right)
$$


where $\mathrm{w}_{\mathrm{a}}\left(\overrightarrow{\mathrm{r}}_{1}\right)$ is the unperturbed wave function for the atom and $\phi_{\alpha}\left(\overrightarrow{\mathrm{r}}_{2}\right)=\exp$ $\left(\mathrm{ik}_{\alpha} \cdot \overrightarrow{\mathrm{r}}_{2}\right)$ is the free particle wave function for the incident dyon.

$$
\mathrm{E}_{\alpha \mathrm{a}}=\mathrm{E}_{\alpha}+\epsilon_{\mathrm{a}}
$$

where, $E_{\alpha}$ is the kinetic energy of the incident dyon and $\epsilon_{a}$ is the unperturbed eigenvalues of the atom.

We can write the wave function $\psi_{\alpha a}^{(+)}$of the total Hamiltonian $\hat{\mathrm{H}}$ in the Born approximation as follows

$$
\begin{array}{r}
\psi_{\alpha \mathrm{a}}^{(+)}\left(\overrightarrow{\mathrm{r}}_{1}, \overrightarrow{\mathrm{r}}_{2}\right)=\phi_{\alpha \mathrm{a}}\left(\overrightarrow{\mathrm{r}}_{1}, \overrightarrow{\mathrm{r}}_{2}\right)+\frac{2 \mathrm{~m}}{\hbar^{2}} \int \mathrm{G}_{\alpha \mathrm{a}}^{(+)}\left(\overrightarrow{\mathrm{r}}_{1}, \overrightarrow{\mathrm{r}}_{2}, \overrightarrow{\mathrm{r}}_{1}^{\prime}, \overrightarrow{\mathrm{r}}_{2}^{\prime}\right) \\
\mathrm{H}^{\prime}\left(\overrightarrow{\mathrm{r}}_{1}^{\prime}, \overrightarrow{\mathrm{r}}_{2}{ }^{\prime}\right) \psi_{\alpha \mathrm{a}}^{(+)}\left(\overrightarrow{\mathrm{r}}_{1}{ }^{\prime}, \overrightarrow{\mathrm{r}}_{2}{ }^{\prime}\right) \mathrm{d}^{3} \mathrm{r}_{1}^{\prime} \mathrm{d}^{3} \mathrm{r}_{2}^{\prime}
\end{array}
$$

where $G_{\alpha a}^{(+)}\left(\vec{r}_{1}, \vec{r}_{2} ; \vec{r}_{1}^{\prime}, \vec{r}_{2}^{\prime}\right)$ is the Green's function for the solution (2.6).

If the yelocity of incident dyon is very high, we can use the Born Approximation (replacing $\psi_{\alpha a}^{(+)}$inside in eqn. (2.7) by the free particle function $\phi_{\alpha \mathrm{a}}$, ) and hence, we can write the asymptotic behaviour of $\psi_{\alpha a}^{(+)}$as follows

$$
\psi_{\alpha \mathrm{a}}^{(+)}\left(\overrightarrow{\mathrm{r}}_{1}, \overrightarrow{\mathrm{r}}_{2}\right) \underset{\mathrm{r}_{2} \rightarrow \infty}{\longrightarrow} \phi_{\alpha \mathrm{a}}\left(\overrightarrow{\mathrm{r}}_{1}, \overrightarrow{\mathrm{r}}_{2}\right)+\sum_{\mathrm{b}} \frac{\exp \left(\mathrm{ik}_{\alpha} \mathrm{r}_{2}\right)}{\mathrm{r}_{2}} \mathrm{f}\left(\overrightarrow{\mathrm{k}}_{\beta}, \mathrm{b} ; \overrightarrow{\mathrm{k}}_{\alpha}, \mathrm{a}\right) \mathrm{w}_{\mathrm{a}}\left(\overrightarrow{\mathrm{r}}_{1}\right)
$$

where

$$
\begin{array}{r}
\mathrm{f}\left(\overrightarrow{\mathrm{k}}_{\beta}, \mathrm{b} ; \overrightarrow{\mathrm{k}}_{\alpha}, \mathrm{a}\right)=-\frac{1}{4 \pi} \cdot \frac{2 \mathrm{~m}}{\hbar^{2}} \iint \exp \left(\mathrm{ik}_{\beta} \cdot \overrightarrow{\mathrm{r}}_{2}^{\prime}\right) \mathrm{w}_{\mathrm{b}}^{*}\left(\overrightarrow{\mathrm{r}}_{1}^{\prime}\right) \mathrm{H}^{\prime}\left(\overrightarrow{\mathrm{r}}_{1}^{\prime}, \overrightarrow{\mathrm{r}}_{2}^{\prime}\right) \\
\phi_{\alpha \mathrm{a}}\left(\overrightarrow{\mathrm{r}}_{1}^{\prime}, \overrightarrow{\mathrm{r}}_{2}^{\prime}\right) \mathrm{d}^{3} \mathrm{r}_{1}^{\prime} \mathrm{d}^{3} \mathrm{r}_{2}^{\prime} \\
=-\frac{2 \mathrm{~m}}{4 \pi \hbar^{2}} \iint \exp \left(-\mathrm{i} \overrightarrow{\mathrm{k}}_{\beta} \cdot \overrightarrow{\mathrm{r}}_{2}^{\prime}\right) \mathrm{w}_{\mathrm{b}}^{*}\left(\overrightarrow{\mathrm{r}}_{1}^{\prime}\right) \mathrm{H}^{\prime}\left(\overrightarrow{\mathrm{r}}_{1}^{\prime}, \overrightarrow{\mathrm{r}}_{2}^{\prime}\right) \\
\exp \left(\overrightarrow{\mathrm{ik}}_{\alpha} \cdot \overrightarrow{\mathrm{r}}_{2}^{\prime}\right) \mathrm{w}_{\mathrm{a}}\left(\overrightarrow{\mathrm{r}}_{1}^{\prime}\right) \mathrm{d}^{3} \mathrm{r}_{1}^{\prime} \mathrm{d}^{3} \mathrm{r}_{2}^{\prime}
\end{array}
$$




$$
\begin{array}{r}
\text { or } \quad \mathrm{f}\left(\overrightarrow{\mathrm{k}}_{\beta}, \mathrm{b} ; \overrightarrow{\mathrm{k}}_{\alpha}, \mathrm{a}\right)=-\frac{2 \mathrm{~m}}{4 \pi \hbar^{2}} \iint \exp \left(\mathrm{i} \overrightarrow{\mathrm{k}} \cdot \overrightarrow{\mathrm{r}}_{2}\right) \mathrm{w}_{\mathrm{b}}^{*}\left(\overrightarrow{\mathrm{r}}_{1}\right) \mathrm{H}^{\prime}\left(\overrightarrow{\mathrm{r}}_{1}^{\prime}, \overrightarrow{\mathrm{r}}_{2}^{\prime}\right) \\
\mathrm{w}_{\mathrm{a}}\left(\overrightarrow{\mathrm{r}}_{1}^{\prime}\right) \mathrm{d}^{3} \mathrm{r}_{1}^{\prime} \mathrm{d}^{3} \mathrm{r}_{2}^{\prime}
\end{array}
$$

where $\quad \overrightarrow{\mathrm{K}}=\left(\overrightarrow{\mathrm{k}}_{\alpha}-\overrightarrow{\mathrm{k}}_{\beta}\right)$

or, deleting primes on the variables of integrations, this can also be written as:

$$
\begin{array}{r}
f\left(\vec{k}_{\beta}, b ; \vec{k}_{\alpha}, a\right)=-\frac{2 m}{4 \pi \hbar^{2}} \iint \exp \left(i \vec{k} \cdot \vec{r}_{2}\right) w_{b}^{*}\left(\vec{r}_{1}\right) H^{\prime}\left(\vec{r}_{1}, \vec{r}_{2}\right) \\
w_{a}\left(\vec{r}_{1}\right) d^{3} r_{1} d^{3} r_{2}
\end{array}
$$

Now, for considering dyon scattering from a Hydrogen atom we start with the following Hamiltonian

$$
\mathrm{H}^{\prime}\left(\overrightarrow{\mathrm{r}}_{1}, \overrightarrow{\mathrm{r}}_{2}\right)=-\frac{\mathrm{e}^{2}}{\mathrm{r}_{12}}+\frac{(\mathrm{eg})^{2}}{2 \mathrm{mr}_{12}^{2}}-\frac{\mathrm{e}^{2}}{\mathrm{r}_{2}}+\frac{(\mathrm{eg})^{2}}{2 \mathrm{Mr}_{2}^{2}}
$$

Thus the scattering amplitude (2.11) can be written as

$$
\begin{gathered}
\mathrm{f}=-\frac{2 \mathrm{~m}}{4 \pi \hbar^{2}} \iint \exp \left(\overrightarrow{\mathrm{k}} \cdot \overrightarrow{\mathrm{r}}_{2}\right)\left[-\mathrm{e}^{2}\left\{\frac{1}{\mathrm{r}_{12}}+\frac{1}{\mathrm{r}_{2}}\right\}+\frac{(\mathrm{eg})^{2}}{2 \mathrm{M}}\left\{\frac{1}{\mathrm{r}_{12}^{2}}+\frac{1}{\mathrm{r}_{2}^{2}}\right\}\right] \\
\mathrm{w}_{\mathrm{b}}^{*}\left(\overrightarrow{\mathrm{r}}_{1}\right) \mathrm{w}_{\mathrm{a}}\left(\overrightarrow{\mathrm{r}}_{1}\right) \mathrm{d}^{3} \mathrm{r}_{1} \mathrm{~d}^{3} \mathrm{r}_{2}
\end{gathered}
$$

Solving this equation in the usual way, we get

$$
\begin{gathered}
\mathrm{f}=\frac{2 \mathrm{me} \mathrm{e}^{2}}{\hbar^{2} \mathrm{k}^{2}} \int\left[1-\exp \left(\mathrm{ik} \cdot \overrightarrow{\mathrm{r}}_{1}\right)\right] \mathrm{w}_{\mathrm{b}}^{*}\left(\overrightarrow{\mathrm{r}}_{1}\right) \mathrm{w}_{\mathrm{a}}\left(\overrightarrow{\mathrm{r}}_{1}\right) \mathrm{d}^{3} \mathrm{r}_{1} \\
+\frac{\mathrm{m}(\mathrm{eg})^{2}}{\mathrm{M} \hbar^{2} \mathrm{k}} \int\left[1-\exp \left(\mathrm{i} \overrightarrow{\mathrm{k}} \cdot \overrightarrow{\mathrm{r}}_{1}\right)\right] \mathrm{w}_{\mathrm{b}}^{*}\left(\overrightarrow{\mathrm{r}}_{1}\right) \mathrm{w}_{\mathrm{a}}\left(\overrightarrow{\mathrm{r}}_{1}\right) \mathrm{d}^{3} \mathrm{r}_{1}
\end{gathered}
$$

For elastic scattering, the initial and final state of the atom are the same i.e. $\mathrm{w}_{\mathrm{a}} \equiv \mathrm{w}_{\mathrm{b}}$ and $\left|\overrightarrow{\mathrm{k}}_{\alpha}\right|=\left|\overrightarrow{\mathrm{k}}_{\beta}\right|$ follows from the energy conservation. Therefore, we may write scattering amplitude as 


$$
\begin{aligned}
\mathrm{f}_{\mathrm{el}} & =\frac{2 \mathrm{me} \mathrm{e}^{2}}{\hbar^{2} \mathrm{k}^{2}} \int\left[1-\exp \left(\mathrm{i} \overrightarrow{\mathrm{k}} \cdot \overrightarrow{\mathrm{r}}_{1}\right)\right] \mathrm{w}_{\mathrm{a}}^{*}\left(\overrightarrow{\mathrm{r}}_{1}\right) \mathrm{w}_{\mathrm{a}}\left(\overrightarrow{\mathrm{r}}_{1}\right) \mathrm{d}^{3} \mathrm{r}_{1} \\
& +\frac{\mathrm{m}(\mathrm{eg})^{2}}{\mathrm{M} \hbar^{2} \mathrm{k}}\left[1-\exp \left(\mathrm{i} \overrightarrow{\mathrm{k}} \cdot \overrightarrow{\mathrm{r}}_{1}\right)\right] \mathrm{w}_{\mathrm{a}}^{*}\left(\overrightarrow{\mathrm{r}}_{1}\right) \mathrm{w}_{\mathrm{a}}\left(\overrightarrow{\mathrm{r}}_{1}\right) \mathrm{d}^{3} \mathrm{r}_{1}
\end{aligned}
$$

If we consider ground state of the atom the scattering cross section is given as $\backslash$

$$
\mathrm{f}_{\mathrm{el}}(\theta)=\frac{2 \mathrm{~m}}{\hbar^{2} \mathrm{k}^{2}}\left[1-\left(1+\frac{1}{4} \mathrm{a}_{\mathrm{o}}^{2} \mathrm{k}^{2}\right)^{-2}\right]\left[\mathrm{e}^{2}+\frac{\mathrm{k}(\mathrm{eg})^{2}}{2 \mathrm{M}}\right]
$$

In the high energy case where Born approximation is valid the scattering amplitude is

$$
\mathrm{f}_{\mathrm{el}} \underset{\mathrm{K} \text { l arg e }}{\longrightarrow} \frac{2 \mathrm{~m}}{\hbar^{2} \mathrm{k}^{2}}\left[\mathrm{e}^{2}+\frac{\mathrm{k}(\mathrm{eg})^{2}}{2 \mathrm{M}}\right]
$$

For inelastic case, $\mathrm{w}_{\mathrm{a}}$ is different from $\mathrm{w}_{\mathrm{b}}$ and hence the first term of both the integrals in equation (2.14) are zero because of orthogonality of unperturbed states of atom. So, we have

$$
\begin{aligned}
\mathrm{f}_{\text {inel. }}= & -\frac{2 \mathrm{me}^{2}}{\hbar^{2} \mathrm{k}^{2}} \int \exp \left(\mathrm{ik} \cdot \overrightarrow{\mathrm{r}}_{1}\right) \mathrm{w}_{\mathrm{b}}^{*}\left(\overrightarrow{\mathrm{r}}_{1}\right) \mathrm{w}_{\mathrm{a}}\left(\overrightarrow{\mathrm{r}}_{1}\right) \mathrm{d}^{3} \mathrm{r}_{1} \\
& -\frac{\mathrm{m}(\mathrm{eg})^{2}}{\mathrm{M} \hbar^{2} \mathrm{k}^{2}} \int \exp \left(\mathrm{ik} \cdot \overrightarrow{\mathrm{r}}_{1}\right) \mathrm{w}_{\mathrm{b}}^{*}\left(\overrightarrow{\mathrm{r}}_{1}\right) \mathrm{w}_{\mathrm{a}}\left(\overrightarrow{\mathrm{r}}_{1}\right) \mathrm{d}^{3} \mathrm{r}_{1}
\end{aligned}
$$

Let us consider the case when the ground state of atom ( $1 \mathrm{~s}$ - state) is excited to the $2 \mathrm{~s}$ state (first excitation) due to collision with incident dyon. The scattering amplitude for this scattering is given by

$$
\mathrm{f}_{\text {inel }}(1 \mathrm{~s} \rightarrow 2 \mathrm{~s})=-\frac{2 \mathrm{~m}}{\hbar^{2} \mathrm{k}^{2}}\left[\mathrm{e}^{2}+\frac{\mathrm{k}(\mathrm{eg})^{2}}{2 \mathrm{M}}\right] \int \exp \left(\overrightarrow{\mathrm{ik}} \cdot \overrightarrow{\mathrm{r}}_{1}\right) \mathrm{w}_{2 \mathrm{~s}}^{*}\left(\overrightarrow{\mathrm{r}}_{1}\right) \mathrm{w}_{1 \mathrm{~s}}\left(\overrightarrow{\mathrm{r}}_{1}\right) \mathrm{d}^{3} \mathrm{r}_{1}
$$


For Hydrogen atom we have

$$
\left.\begin{array}{l}
\mathrm{w}_{1 \mathrm{~s}}=\frac{1}{\sqrt{\pi \mathrm{a}_{0}^{3}}} \exp \left(-\mathrm{r} / \mathrm{a}_{0}\right) \\
\mathrm{w}_{2 \mathrm{~s}}=\frac{1}{4 \sqrt{2 \pi \mathrm{a}_{0}^{3}}}\left(2-\mathrm{r} / \mathrm{a}_{0}\right) \exp \left(-\mathrm{r} / \mathrm{a}_{0}\right)
\end{array}\right\}
$$

where $\mathrm{a}_{\mathrm{o}}=\frac{\hbar^{2}}{\mathrm{Me}^{2}}$

There fore,

$$
\begin{aligned}
\mathrm{f}_{\text {inel }}(1 \mathrm{~s} \rightarrow 2 \mathrm{~s})=-\frac{2 \mathrm{~m}}{\hbar^{2} \mathrm{k}^{2}}\left[\left\{\mathrm{e}^{2}+\frac{\mathrm{k}(\mathrm{eg})^{2}}{2 \mathrm{M}}\right\} \frac{1}{4 \pi \sqrt{2} \mathrm{a}_{\mathrm{o}}^{3}}\right] \\
\qquad \exp (\mathrm{i} \overrightarrow{\mathrm{k}} \cdot \overrightarrow{\mathrm{r}}) \exp \left(2-\frac{\mathrm{r}}{\mathrm{a}_{\mathrm{o}}}\right) \exp \left(-\frac{3}{2} \frac{\mathrm{r}}{\mathrm{a}_{\mathrm{o}}}\right) \mathrm{d}^{3} \mathrm{r}
\end{aligned}
$$

The above equation gives us

$$
\mathrm{f}_{\text {inel }}=\frac{-8 \sqrt{2} \cdot \mathrm{a}_{\mathrm{o}}^{2}}{\hbar^{2}\left(\frac{9}{4}+\mathrm{k}^{2} \mathrm{a}_{\mathrm{o}}^{2}\right)^{3}}\left[\mathrm{e}^{2}+\frac{\mathrm{k}(\mathrm{eg})^{2}}{2 \mathrm{M}}\right]
$$

\section{EXCHANGE SCATTERING OF DYON FROM HYDROGEN ATOM}

In exchange scattering, the dyon takes the place of electron of the atom (having coordinate $r_{1}$ ) and the electron of the atom is scattered. In this case, coordinate of the incident dyon $r_{2}$ and coordinate of the electron of the atom (as shown in fig. 1) i.e. $r_{2}$ are interchanged after scattering has taken place and hence the final state $\phi_{\beta b}$ for the exchange scattering can be written as

$$
\phi_{\beta \mathrm{b}}^{\text {exch. }}\left(\overrightarrow{\mathrm{r}}_{1}, \overrightarrow{\mathrm{r}}_{2}\right)=\exp \left[\mathrm{ik}_{\beta} \cdot \mathrm{r}_{1}\right] \mathrm{w}_{\mathrm{b}}\left(\mathrm{r}_{2}\right)
$$


Also, the asymptotic behaviour of $\psi_{\alpha \mathrm{a}}^{(+)}\left(\overrightarrow{\mathrm{r}}_{1}, \overrightarrow{\mathrm{r}}_{2}\right)$ is given by

$$
\psi_{\alpha \mathrm{a}}^{(+)}\left(\overrightarrow{\mathrm{r}}_{1}, \overrightarrow{\mathrm{r}}_{2}\right) \underset{\mathrm{r}}{\mathrm{r}_{1} \rightarrow \infty} \sum_{\mathrm{b}} \frac{\exp \left(\mathrm{ik}_{\beta} \mathrm{r}_{1}\right)}{\mathrm{r}_{1}} \mathrm{~g}\left(\overrightarrow{\mathrm{k}}_{\beta}, \mathrm{b} ; \overrightarrow{\mathrm{k}}_{\alpha}, \mathrm{a}\right) \mathrm{w}_{\mathrm{b}}\left(\overrightarrow{\mathrm{r}}_{2}\right)
$$

There is no scattered part here because the dyon is captured in the atom. The scattering amplitude $g\left(\overrightarrow{\mathrm{k}}_{\beta}, \mathrm{b} ; \overrightarrow{\mathrm{k}}_{\alpha}\right.$, a) in the exchange scattering is given by

$$
\begin{aligned}
g\left(\overrightarrow{\mathrm{k}}_{\beta}, \mathrm{b} ; \overrightarrow{\mathrm{k}}_{\alpha}, \mathrm{a}\right) & =-\frac{2 \mathrm{~m}}{4 \pi \hbar^{2}} \iint \phi_{\beta \mathrm{b}}^{\text {exch.* }}\left(\overrightarrow{\mathrm{r}}_{1}, \overrightarrow{\mathrm{r}}_{2}\right) \mathrm{H}^{\prime}\left(\overrightarrow{\mathrm{r}}_{1}, \overrightarrow{\mathrm{r}}_{2}\right) \psi_{\alpha \mathrm{a}}^{(+)}\left(\overrightarrow{\mathrm{r}}_{1}, \overrightarrow{\mathrm{r}}_{2}\right) \mathrm{d}^{3} \mathrm{r}_{1} \mathrm{~d}^{3} \mathrm{r}_{2} \\
& =-\frac{2 \mathrm{~m}}{4 \pi \hbar^{2}} \iint \exp \left(\overrightarrow{\mathrm{ik}}_{\beta} \cdot \mathrm{r}_{1}\right) \mathrm{w}_{\mathrm{a}}^{*}\left(\mathrm{r}_{2}\right) \mathrm{H}^{\prime}\left(\overrightarrow{\mathrm{r}}_{1}, \overrightarrow{\mathrm{r}}_{2}\right) \psi_{\alpha \mathrm{a}}^{(+)}\left(\overrightarrow{\mathrm{r}}_{1}, \overrightarrow{\mathrm{r}}_{2}\right) \mathrm{d}^{3} \mathrm{r}_{1} \mathrm{~d}^{3} \mathrm{r}_{2}
\end{aligned}
$$

Since, we are assuming that the incident dyon is a fermion so that total wavefunction must be antisymmetric. In non-relativistic limit the wavefunction can be written as follows

$$
\psi^{(+)}\left(\overrightarrow{\mathrm{r}}_{1}, \overrightarrow{\mathrm{r}}_{2} ; \overrightarrow{\mathrm{s}}_{1}, \overrightarrow{\mathrm{s}}_{2}\right)=\psi^{(+)}\left(\overrightarrow{\mathrm{r}}_{1}, \overrightarrow{\mathrm{r}}_{2}\right) \chi^{(+)}\left(\overrightarrow{\mathrm{s}}_{1}, \overrightarrow{\mathrm{s}}_{2}\right)
$$

where $\psi^{(+)}\left(\vec{r}_{1}, \vec{r}_{2}\right)$ is the space part and $\chi^{(+)}\left(\vec{s}_{1}, \vec{s}_{2}\right)$ is spin part of the total wave function. Since $\chi^{4}\left(\vec{s}_{1}, \vec{s}_{2}\right)$ describes two spin half particles, we can have either a singlet state or a triplet state. The singlet state is

$$
\chi_{\text {sin g. }}^{(+)}\left(\vec{s}_{1}, \vec{s}_{2}\right)=\frac{1}{\sqrt{2}}\{\alpha(1) \beta(2)-\alpha(2) \beta(1)\}
$$

which is antisymmetric. Thus to make the total wave function antisymmetric, we symmetrize the space part

$$
\psi_{\text {sym. }}^{(+)}\left(\vec{r}_{1}, \vec{r}_{2}\right)=\psi_{\alpha a}^{(+)}\left(\vec{r}_{1}, \vec{r}_{2}\right)+\psi_{\alpha a}^{(+)}\left(\vec{r}_{2}, \vec{r}_{1}\right)
$$

The first function on the right hand side corresponds to the direct scattering and the second one corresponds to exchange scattering. Asymptotic behavior of the symmetrized wavefunction (3.6) can be written as

$\psi_{\text {sym. }}^{(+)}\left(\vec{r}_{1}, \vec{r}_{2}\right) \underset{r_{1} \rightarrow \infty}{\longrightarrow} \phi_{\alpha a}\left(\vec{r}_{1}, \vec{r}_{2}\right)+\sum_{b} \frac{\exp \left(\mathrm{ik}_{\beta} \mathrm{r}_{1}\right)}{r_{1}}(f+g) w_{b}\left(\vec{r}_{2}\right)$ 
Thus the scattering amplitude in the singlet state is the sum of the direct and exchange scattering amplitudes and hence the scattering cross section for singlet state is given by

$$
\sigma_{\text {sin g. }}=\frac{\mathrm{k}_{\beta}}{\mathrm{k}_{\alpha}}|\mathrm{f}+\mathrm{g}|^{2}
$$

The triplet state is given by

$$
\chi_{\text {trip. }}^{(+)}\left(\vec{s}_{1}, \vec{s}_{2}\right)=\left\{\begin{array}{c}
\alpha(1) \alpha(2) \\
\beta(1) \beta(2) \\
\frac{1}{\sqrt{2}}\{\alpha(1) \beta(2)+\alpha(2) \beta(1)\}
\end{array}\right.
$$

i.e. all the triplet states are symmetric. Thus to make the total wave function antisymmetric, we make the space part antisymmetric

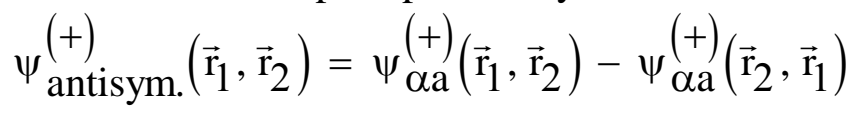

In this case, scattering amplitude will be $|\mathrm{f}-\mathrm{g}|$ and hence the scattering cross-section in triplet state is given by

$$
\sigma_{\text {trip. }}=\frac{\mathrm{k}_{\beta}}{\mathrm{k}_{\alpha}}|\mathrm{f}-\mathrm{g}|^{2}
$$

Equation (3.8) and (3.11) give the differential scattering cross section including exchange effect. Total differential scattering cross section is the sum of the $\sigma_{\text {sin g. }}$ and $\sigma_{\text {trip. }}$ with their proper statistical weight factor

$$
\left(\frac{\mathrm{d} \sigma}{\mathrm{d} \Omega}\right)_{\text {tot. }}=\frac{1}{4} \sigma_{\text {sin g. }}+\frac{3}{4} \sigma_{\text {trip. }}
$$

In order to evaluate equation (3.12) we should calculate $\mathrm{f}$ and g. The direct scattering amplitude $f$ has already been calculated and is given by equation (2.14) while $g$ is given by

$$
g=-\frac{2 \mathrm{~m}}{4 \pi \hbar^{2}} \iint \exp \left(-\mathrm{i} \vec{k}_{\beta} \cdot \overrightarrow{\mathrm{r}}_{1}\right) \mathrm{w}_{\mathrm{b}}^{*}\left(\overrightarrow{\mathrm{r}}_{2}\right) \mathrm{H}^{\prime}\left(\overrightarrow{\mathrm{r}}_{1}, \overrightarrow{\mathrm{r}}_{2}\right) \psi_{\alpha \mathrm{a}}^{(+)}\left(\overrightarrow{\mathrm{r}}_{1}, \overrightarrow{\mathrm{r}}_{2}\right) \mathrm{d}^{3} \mathrm{r}_{1} \mathrm{~d}^{3} \mathrm{r}_{2}
$$

To calculate g, we make use of Born-Oppenheimer-approximation. In this approximation, we replace $\psi_{\alpha a}^{(+)}\left(\vec{r}_{1}, \vec{r}_{2}\right)$ by the state $\phi_{\alpha a}\left(\vec{r}_{1}, \vec{r}_{2}\right)$; the unperturbed wave function before scattering 


$$
\mathrm{g}=-\frac{1}{4 \pi} \cdot \frac{2 \mathrm{~m}}{\hbar^{2}} \iint \exp \left(-\mathrm{i} \overrightarrow{\mathrm{k}}_{\beta} \cdot \overrightarrow{\mathrm{r}}_{1}\right) \mathrm{w}_{\mathrm{b}}^{*}\left(\overrightarrow{\mathrm{r}}_{2}\right) \mathrm{H}^{\prime}\left(\overrightarrow{\mathrm{r}}_{1}, \overrightarrow{\mathrm{r}}_{2}\right) \exp \left(\overrightarrow{\mathrm{ik}}_{\alpha} \cdot \mathrm{r}_{2}\right) \mathrm{w}_{\mathrm{a}}\left(\overrightarrow{\mathrm{r}}_{1}\right) \mathrm{d}^{3} \mathrm{r}_{1} \mathrm{~d}^{3} \mathrm{r}_{2}
$$

The initial and the final states of the atom are not orthogonal to each other. Due to this, a number of defects are incorporated into Born-Oppenheimer approximation. To overcome these difficulties we use another approximation which is due to Ochkur. In this approximation, $\mathrm{g}$ is expanded in the inverse powers of $\mathrm{k}_{\alpha}$ and the leading term is only retained, We have

$$
\mathrm{g} \equiv \mathrm{g}_{\mathrm{ee}}+\mathrm{g}_{\mathrm{ne}}
$$

Where $\mathrm{g}_{\mathrm{ee}}$ is the contribution due to dyon-electron interaction and $\mathrm{g}_{\mathrm{ne}}$ is the contribution due to nuclear interaction.

We have

$$
\begin{aligned}
\mathrm{g}_{\mathrm{ee}}= & -\frac{2 \mathrm{me}^{2}}{4 \pi \hbar^{2}} \iint \exp \left\{-\mathrm{i} \overrightarrow{\mathrm{k}}_{\beta} \cdot \overrightarrow{\mathrm{r}}_{1}\right\} \mathrm{w}_{\mathrm{b}}^{*}\left(\overrightarrow{\mathrm{r}}_{2}\right) \frac{1}{\mathrm{r}_{12}} \exp \left(\mathrm{ik}_{\alpha} \cdot \overrightarrow{\mathrm{r}}_{2}\right) \mathrm{w}_{\mathrm{a}}\left(\overrightarrow{\mathrm{r}}_{1}\right) \mathrm{d}^{3} \mathrm{r}_{1} \mathrm{~d}^{3} \mathrm{r}_{2} \\
& +\frac{\mathrm{m}(\mathrm{eg})^{2}}{2 \pi \mathrm{M} \hbar^{2}} \iint \exp \left\{-\overrightarrow{\mathrm{i}}_{\beta} \cdot \overrightarrow{\mathrm{r}}_{1}\right\} \mathrm{w}_{\mathrm{b}}^{*}\left(\overrightarrow{\mathrm{r}}_{2}\right) \frac{1}{\mathrm{r}_{12}^{2}} \exp \left(\overrightarrow{\mathrm{ik}}_{\alpha} \cdot \overrightarrow{\mathrm{r}}_{2}\right) \mathrm{w}_{\mathrm{a}}\left(\overrightarrow{\mathrm{r}}_{1}\right) \mathrm{d}^{3} \mathrm{r}_{1} \mathrm{~d}^{3} \mathrm{r}_{2}
\end{aligned}
$$

Now

$$
\frac{1}{\mathrm{r}_{12}}=\frac{1}{2 \pi^{2}} \int \frac{\exp \left(\mathrm{i} \overrightarrow{\mathrm{S}}\left(\overrightarrow{\mathrm{r}}_{2}-\overrightarrow{\mathrm{r}}_{1}\right)\right)}{\mathrm{s}^{2}} \mathrm{~d}^{3} \mathrm{~S}
$$

and hence

$$
\begin{aligned}
\mathrm{g}_{(\text {Ochkur })}= & -\frac{2 \mathrm{me} \mathrm{e}^{2}}{\hbar^{2}} \cdot \frac{1}{\mathrm{~K}_{\alpha}^{2}} \int \exp \left(\overrightarrow{\mathrm{ik}}_{\alpha} \cdot \overrightarrow{\mathrm{r}}_{2}\right) \mathrm{w}_{\mathrm{b}}^{*}\left(\overrightarrow{\mathrm{r}}_{2}\right) \mathrm{w}_{\mathrm{a}}\left(\overrightarrow{\mathrm{r}}_{2}\right) \mathrm{d}^{3} \mathrm{r}_{2} \\
& +\frac{\mathrm{m}(\mathrm{eg})^{2}}{\pi \mathrm{M} \hbar^{2}} \cdot \frac{1}{\mathrm{~K}_{\alpha}} \int \exp \left(\overrightarrow{\mathrm{i}}_{\alpha} \cdot \overrightarrow{\mathrm{r}}_{2}\right) \mathrm{w}_{\mathrm{b}}^{*}\left(\overrightarrow{\mathrm{r}}_{2}\right) \mathrm{w}_{\mathrm{a}}\left(\overrightarrow{\mathrm{r}}_{2}\right) \mathrm{d}^{3} \mathrm{r}_{2}
\end{aligned}
$$

with the help of above equation and from the direct scattering amplitude (2.14). we get

$$
\mathrm{g}_{\text {(Ochkur) }}=\frac{\mathrm{k}^{2}}{\mathrm{k}_{\alpha}^{2}}\left[\mathrm{f}-\frac{2 \mathrm{me}^{2}}{\hbar^{2} \mathrm{k}^{2}} \delta_{\text {ba }}+\frac{\mathrm{m}(\mathrm{eg})^{2}}{\pi \mathrm{M} \hbar^{2}} \cdot \frac{\mathrm{K}_{\alpha}}{\mathrm{K}} \delta_{\text {ba }}\right]
$$


For inelastic scattering $\mathrm{a} \neq \mathrm{b}$. Therefore $\delta_{\mathrm{ab}}=\mathrm{o}$ and hence we get

$$
\mathrm{g}_{(\text {Ochkur })}=\frac{\mathrm{k}^{2}}{\mathrm{~K}_{\alpha}^{2}} \cdot \mathrm{f}
$$

For elastic scattering, $\mathrm{a}=\mathrm{b}$ i.e. $\delta_{\mathrm{ba}}=1$. Therefore

$$
\mathrm{g}_{\text {(Ochkur) }}=\frac{\mathrm{k}^{2}}{\mathrm{k}_{\alpha}^{2}}\left[\mathrm{f}-\frac{2 \mathrm{me}^{2}}{\hbar^{2} \mathrm{k}^{2}}+\frac{\mathrm{m}(\mathrm{eg})^{2}}{\pi \mathrm{M} \hbar^{2}} \cdot \frac{\mathrm{K}_{\alpha}}{\mathrm{K}}\right]
$$

Now, for elastic scattering of dyon with hydrogen atom.

$$
\mathrm{f}=\frac{2 \mathrm{~m}}{\hbar^{2} \mathrm{k}^{2}}\left[1-\left(1+\frac{1}{4} \mathrm{a}_{\mathrm{o}}^{2} \mathrm{k}^{2}\right)^{-2}\right]\left[\mathrm{e}^{2}+\frac{\mathrm{k}(\mathrm{eg})^{2}}{2 \mathrm{M} \pi}\right]
$$

For Born approximation

$$
\mathrm{f}=\frac{2 \mathrm{~m}}{\hbar^{2} \mathrm{k}^{2}}\left[\mathrm{e}^{2}+\frac{\mathrm{k}(\mathrm{eg})^{2}}{\pi \mathrm{M}}\right]
$$

Therefore

$$
g_{(\text {Ochkur })}=\frac{1}{\mathrm{k}_{\alpha}^{2}}\left[\frac{2 \mathrm{~m}(\mathrm{eg})^{2}}{\pi \mathrm{M} \hbar^{2} \mathrm{k}^{2}}\left(1+\frac{\mathrm{K}_{\alpha}}{\mathrm{K}}\right)\right]
$$

Hence the elastic differential scattering cross section for dyon-atom scattering in the exchange effect is given by

$$
\begin{aligned}
\left(\frac{\mathrm{d} \sigma}{\mathrm{d} \Omega}\right)_{\text {tot. }} & =\frac{1}{4}\left|\mathrm{f}+\mathrm{g}_{\text {Ochkur }}\right|^{2}+\frac{3}{4}\left|\mathrm{f}-\mathrm{g}_{\text {Ochkur }}\right|^{2} \\
& =\frac{\mathrm{me}^{2}}{\hbar^{2} \mathrm{k}^{2}}-\frac{\mathrm{m}(\mathrm{eg})^{2}}{\pi \mathrm{M} \hbar^{2}}\left[\frac{\mathrm{K}_{\alpha}-\mathrm{k}^{2}}{\mathrm{KK}_{\alpha}}\right]
\end{aligned}
$$


Fig. 1 Index 'A' refers to incident d yon and indices ' 1 ' and ' 2 ' refers to proton and electron of the Hydrogen atom respectively.

\section{DISCUSSION}

Hamiltonian (3.1) describes the dynamics for scattering of a dyon by a Hydrogen atom. Equation (3.7) is the wave function for the total Hamiltonian under Born approximation and scattering amplitude for this case is given by equation (3.10). Equation (3.16) describes the scattering amplitude under Born approximation for elastic scattering . Scattering amplitude for elastic scattering given by equation (3.17). Equation (4.8) and (4.11) give the differential scattering cross section including exchange effect i.e. where electron of hydrogen atom is replaced by the incident dyon in the scattering process. Total differential scattering cross section is given by equation (4.12). Equation (4.22) describes the total scattering cross section for scattering of a dyon by hydrogen atom with inclusion of exchange effect. All of these scattering cross sections are modified from the usual scattering cross section of scattering of a fermion on the atom due to the presence of magnetic charge on dyon. These scattering cross sections reduce to the usual scattering cross sections of scattering of a fermion from an atom in the absence of magnetic charge.

\section{REFERNCES}

1. P.A.M. Dirac; Proc. R. Soc. London A133 (1931) 60.

2. P.A.M. Dirac; Phys. Rev. 74 (1948) 817.

3. G.'t Hooft; Nucl. Phys. B79 (1974) 276.

4. A. Polyakov; JETP Lett. 20 (1974) 194.

5. S. Mandelstam; Phys. Rep. 23 (1976) 245.

Phys. Rev. $\underline{\mathrm{D} 9}$ (1979) 249. 
6. E. Witten; Phys. Lett. B86 (1979) 283.

7. C.J. Callon Jr.; Phys. Rev. D26 (1982) 2058.

Phys. Rev. D25 (1982) 2141.

V.A. Rubakov; Nucl. Phys. B203(1982) 311.

8. V.A. Rubakov; Sov. Phys. JETP Lett. 33 (1981) 645.

9. A.S. Goldhaber; Phys. Rev. Lett. 36 (1976) 1122.

10. J. Schwinger; Phys. Rev. 114 (1979) 283.

Ann. Phys. 101 (1976) 451.

11. B.S. Rajput and D.C. Joshi; Had. J. $\underline{4}$ (1981) 1805.

B.S. Rajput and Om Prakash; Ind. J. Phys. A53 (1979) 274.

Ind. J. Pure \& Appl. Phys. 16 (1978) 993.

12. B.S. Rajput and D.S. Bhakuni; Lett. Nuovo Cim. 34 (1982) 509.

13. D.S. Bhakuni, O.P.S. Negi and B.S. Rajput; Lett. Nuovo Cim. $\underline{36}$ (1983) 15.

14. V.P. Pandey and B.S.Rajput; A13 (1998) 5245.

15. V.P. Pandey, H.C. Chandola and B.S. Rajput; Il Nuovo Cim. A102 (1990) 1507.

V.P. Pandey and B.S. Rajput; Il Nuovo Cim. 111B (1996) 275.

16. P.C. Pant, V.P. Pandey and B.S. Rajput; Il Nuovo Cim. 110A (1997) 1421.

17. P.C. Pant, V.P. Pandey and B.S. Rajput; Il Nuovo Cim. $\underline{110 \mathrm{~A}}$ (1997) 829.

18. P.C. Pant, V.P. Pandey and B.S. Rajput; Il Nuovo Cim. (Communicated).

19. P.C. Pant, V.P. Pandey and B.S. Rajput; Il Nuovo Cim. (Communicated). 